\title{
Statistics of counter-streaming solar wind suprathermal electrons at solar minimum: STEREO observations
}

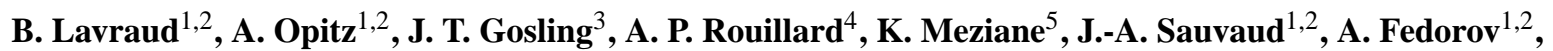

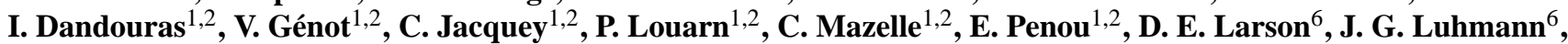 \\ P. Schroeder ${ }^{6}$, L. Jian ${ }^{7}$, C. T. Russell ${ }^{7}$, C. Foullon ${ }^{8}$, R. M. Skoug ${ }^{9}$, J. T. Steinberg ${ }^{9}$, K. D. Simunac ${ }^{10}$, and A. B. Galvin ${ }^{10}$ \\ ${ }^{1}$ Centre d'Etude Spatiale des Rayonnements, Université de Toulouse (UPS), 9 Avenue du Colonel Roche, 31028 Toulouse \\ Cedex 4, France \\ ${ }^{2}$ Centre National de la Recherche Scientifique, UMR 5187, Toulouse, France \\ ${ }^{3}$ Laboratory for Atmospheric and Space Physics, University of Colorado, Boulder, CO, USA \\ ${ }^{4}$ Space Environment Physics Group, School of Physics and Astronomy, University of Southampton, UK \\ ${ }^{5}$ Department of Physics, University of New Brunswick, Fredericton, Canada \\ ${ }^{6}$ Space Sciences Laboratory, University of California, Berkeley, USA \\ ${ }^{7}$ Institute of Geophysics and Planetary Physics, University of California, Los Angeles, USA \\ ${ }^{8}$ Centre for Fusion, Space and Astrophysics, Department of Physics, University of Warwick, Coventry, UK \\ ${ }^{9}$ Space Science and Applications, Los Alamos National Laboratory, Los Alamos, New Mexico, USA \\ ${ }^{10}$ University of New Hampshire, Durham, USA
}

Received: 6 August 2009 - Revised: 10 December 2009 - Accepted: 18 January 2010 - Published: 22 January 2010

\begin{abstract}
Previous work has shown that solar wind suprathermal electrons can display a number of features in terms of their anisotropy. Of importance is the occurrence of counter-streaming electron patterns, i.e., with "beams" both parallel and anti-parallel to the local magnetic field, which is believed to shed light on the heliospheric magnetic field topology. In the present study, we use STEREO data to obtain the statistical properties of counter-streaming suprathermal electrons (CSEs) in the vicinity of corotating interaction regions (CIRs) during the period March-December 2007. Because this period corresponds to a minimum of solar activity, the results are unrelated to the sampling of large-scale coronal mass ejections, which can lead to CSE owing to their closed magnetic field topology. The present study statistically confirms that CSEs are primarily the result of suprathermal electron leakage from the compressed CIR into the upstream regions with the combined occurrence of halo depletion at $90^{\circ}$ pitch angle. The occurrence rate of CSE is found to be about $15-20 \%$ on average during the period analyzed (depending on the criteria used), but superposed epoch analysis demonstrates that CSEs are preferentially observed both before and after the passage of the stream interface
\end{abstract}

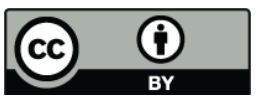

Correspondence to: B. Lavraud (benoit.lavraud@cesr.fr) (with peak occurrence rate $>35 \%$ in the trailing high speed stream), as well as both inside and outside CIRs. The results quantitatively show that CSEs are common in the solar wind during solar minimum, but yet they suggest that such distributions would be much more common if pitch angle scattering were absent. We further argue that (1) the formation of shocks contributes to the occurrence of enhanced counter-streaming sunward-directed fluxes, but does not appear to be a necessary condition, and (2) that the presence of small-scale transients with closed-field topologies likely also contributes to the occurrence of counter-streaming patterns, but only in the slow solar wind prior to CIRs.

Keywords. Interplanetary physics (Energetic particles; Interplanetary shocks) - Ionosphere (Wave-particle interactions)

\section{Introduction}

\subsection{Counter-streaming suprathermal electron patterns}

Owing to their mean-free path of order $1 \mathrm{AU}$, solar wind suprathermal electrons are essentially collision-less. They travel freely along the magnetic field while thermal electrons and ions are essentially bound to one another in the solar wind rest frame. Up to about $2 \mathrm{keV}$, solar wind electrons can

Published by Copernicus Publications on behalf of the European Geosciences Union. 
Back scattered halo

Mirrored back scattered halo

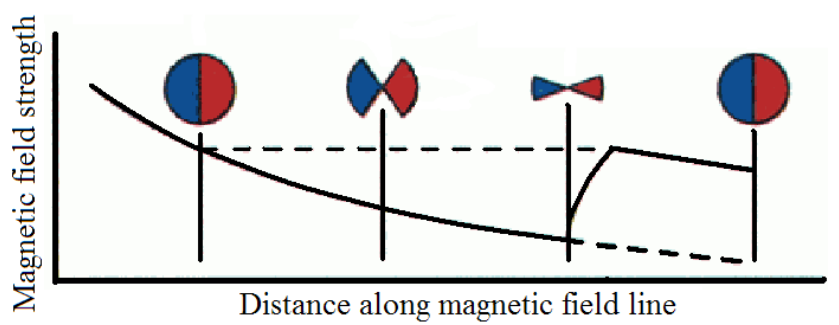

Fig. 1. Schematic illustration of changes in suprathermal electron pitch angle distributions assuming adiabatic evolution along a magnetic field line with a non-monotonic magnetic strength decrease away from the Sun. See text for details. Adapted from Gosling et al. (2001a) and Skoug et al. (2005).

be decomposed into three main populations (e.g., Feldman et al., 1975; Pilipp et al., 1987a): (1) the "core" population, extending from zero to several tens of $\mathrm{eV}$ and whose density, temperature and anisotropy are variable; (2) the "halo" population, a tenuous and generally isotropic suprathermal population (typically above $70 \mathrm{eV}$ ); and (3) the "strahl", an intense beam of suprathermal electrons aligned to the magnetic field and directed outward from the Sun; it carries a substantial heat flux away from the solar corona. Solar wind electron spectra near $1 \mathrm{AU}$ are characterized by a clear spectral breakpoint around $70 \mathrm{eV}$ that separates the halo and strahl from the thermal electrons.

There often occur substantial departures from the general characteristics of solar wind electrons given above. This is specifically true for the suprathermal populations (halo and strahl) whose properties shed light on the heliospheric magnetic topology (e.g., Gosling et al., 1987; Gosling, 1990; Kahler and Lin, 1994). In particular, counter-streaming, i.e., sunward-directed suprathermal electron beam-like features are frequently observed in the solar wind.

1. Strahl broadening at heliospheric distances beyond 1 AU (Hammond et al., 1996) may occur due to waveparticle interactions and pitch angle (PA) scattering (e.g., Gary et al., 1994; Vocks et al., 2005; Saito and Gary, 2007; de Koning et al., 2007; Owens et al., 2008, and references therein). This process is also deemed to contribute to the formation of the sunward-directed portion of the roughly isotropic halo population (Gosling et al., 2001a; Maksimovic et al., 2005; Štverák et al., 2009), which cannot be reproduced by simple exospheric solar wind models (Lie-Svendsen et al., 1997; Pierrard et al., 1999; Maksimovic et al., 2005).

2. Counter-streaming beam patterns may occur owing to the presence of magnetic field enhancements, e.g., within corotating interaction regions and at their bounding shocks (CIR; cf. Pizzo, 1978; Gosling and Pizzo, 1999), downstream along magnetic field lines from the observation point. The backscattering there likely results from wave-particle interactions and shock heating combined with simple adiabatic mirroring and particle leakage into the upstream regions of the CIR (Gosling et al., 1993; Steinberg et al., 2005; Skoug et al., 2006). This is discussed in further details in Sect. 4.

3. Another feature of suprathermal solar wind electrons are halo depletions at $90^{\circ}$ PA (Gosling et al., 2001a, 2002; Skoug et al., 2006). From visual inspection, Gosling et al. (2001a) found that about $10 \%$ of the $272 \mathrm{eV}$ electron PA distributions measured by ACE at 1 AU show a significant depletion in phase space density (PSD) centered at $90^{\circ}$. They explained such depletions as the result of adiabatic mirroring and focusing of suprathermal electrons when a magnetic field enhancement is present farther out along the magnetic field line. The PA depletion width at a given observation point is then related to the ratio of the maximum field strength farther out to that measured locally (cf. Fig. 1). Unlike CIR-leakage and shock-related counterstreaming electron patterns (cf. point (2) above and Sect. 4 later), which lead to an enhancement in the PSD of the counter-streaming part of the population, the apparent counter-streaming that results from such depletions of the halo population is deemed to come from an actual decrease in PSD centered at $90^{\circ}$ owing to conservation of the first adiabatic invariant. Differentiation between the two processes is sometimes difficult because both effects can be present simultaneously (Steinberg et al., 2005).

4. Subject to less controversy, counter-streaming strahls are also observed within closed solar wind field lines with both ends attached to the Sun, such as within interplanetary coronal mass ejections (ICMEs) (e.g., Zwickl et al., 1983; Pilipp et al., 1987b; Gosling et al., 1987, 2002, Gosling, 1990) and newly closed field regions following from magnetic reconnection in the solar wind at the heliospheric current sheet (HCS) (Gosling et al., 2006; Lavraud et al., 2009) (see also Onsager et al., 2001, and Lavraud et al., 2006, for a similar concept applied to the magnetosphere).

\subsection{Structure of corotating interaction regions}

Corotating interaction regions (CIRs; cf. Fig. 2) are due to the overtaking (e.g., Sarabhai, 1963; Neugebauer and Snyder, 1966; Carovillano and Siscoe, 1969) of the slow solar wind from the vicinity of the heliospheric current sheet (HCS) (Schulz, 1973) by the fast solar wind that emanates from the adjacent trailing coronal hole (e.g., Gosling and Pizzo, 1999, and references therein). The HCS is typically embedded within slow and dense solar wind, forming a region generally called the heliospheric plasma sheet (in analogy to that in Earth's magnetospheric tail) (e.g., Burlaga et al., 1990; 
Winterhalter et al., 1994). The slow solar wind that is sunward of the HCS is what directly interacts with the trailing fast solar wind (although the HCS is often already caught up by the CIR at $1 \mathrm{AU}$ ). Both these winds usually have the same magnetic field polarity since they are on the same side of the HCS. The boundary between the slow and fast solar winds is termed the stream interface (SI) (Burlaga, 1974; Gosling et al., 1978). As depicted in Fig. 2, this interaction leads to the formation of a compression region (grey area in Fig. 2) with enhanced densities and magnetic field strengths (e.g., Pizzo, 1978; Gosling and Pizzo, 1999). Depending on the local plasma properties and geometry of the magnetic field and flow, forward and reverse shocks may form ahead and behind the compression region (long edges of the grey area in Fig. 2). These typically form beyond 1 AU (Hundhausen, 1973). The magnetic field lines that thread the compression region extend "upstream" into both the uncompressed slow and fast solar wind. This leads to a magnetic strength profile along magnetic field lines threading a CIR that resembles that sketched in Fig. 1. This topology is expected on both sides of the CIR.

In the present paper, we investigate the properties of solar wind suprathermal electron PA distributions using STEREO data during 1 March-31 December 2007. Under the prevalent solar minimum conditions of this period, the processes identified in Sect. 1.1 as responsible for counter-streaming suprathermal electron patterns are deemed to be related primarily to the nearby presence of structures such as CIRs and shocks. Previous work on counter-streaming electrons associated with CIRs and shocks have been largely based on a few case studies (Gosling et al., 2001a, 2002; Steinberg et al., 2005; Skoug et al., 2006). The aim of the present statistical study is to (1) quantify the occurrence rate of CSE as a function of definition criteria, (2) determine the CSE occurrence rate as a function of location relative to CIRs in order to demonstrate the aforementioned relationship, and (3) investigate the potential roles of pitch angle scattering and the presence of CIR-bounding shocks.

In the following, we define as "counter-streaming electron" (CSE) events those PA distributions that show higher phase space densities (PSDs) in both the field-aligned and anti-field-aligned directions as compared to the perpendicu$\operatorname{lar}\left(90^{\circ} \mathrm{PA}\right)$ direction. Section 2 presents the data used in the study. In Sect. 3 we use an event to illustrate the occurrence of CSE patterns, and then perform statistical analyses of the dataset. We discuss the results in Sect. 4 and give the conclusions in Sect. 5.

\section{Instrumentation}

The Solar Terrestrial Relations Observatory (STEREO; Kaiser et al., 2008) mission consists of two spacecraft that slowly drift ahead (referred to as ST-A) and behind (referred to as ST-B) the Earth on similar orbits around the Sun. The

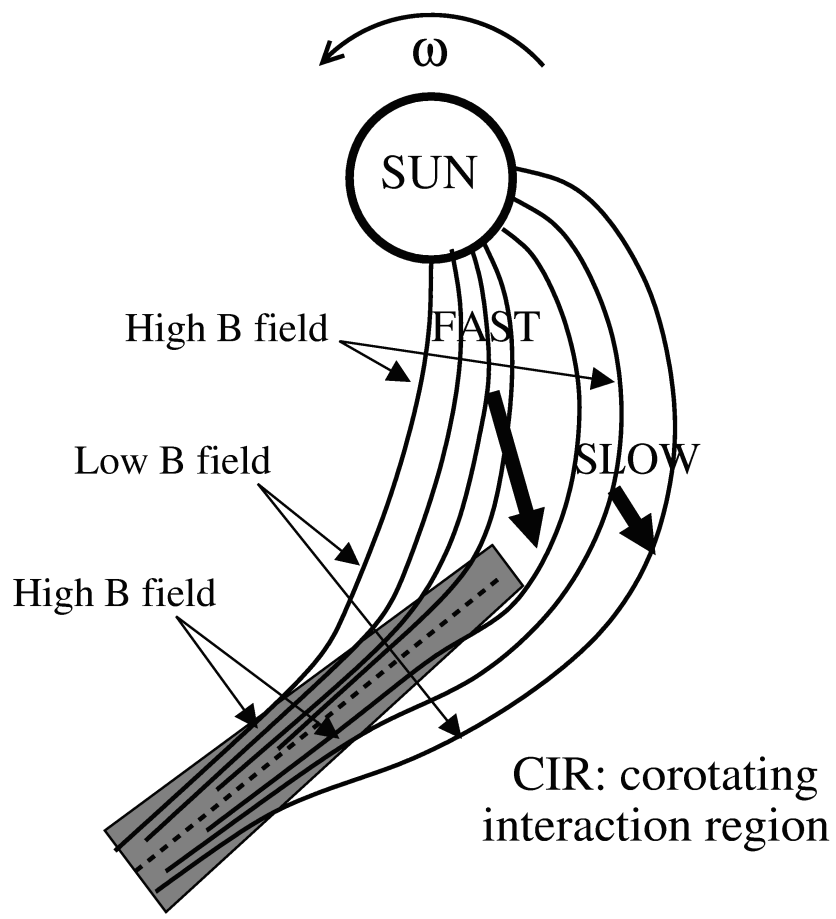

Fig. 2. Schematic of the structure of a corotating interaction region (CIR) in the solar wind which may lead to (1) magnetic field strength profiles, along a given field line, like that depicted in Fig. 1 in both the high-speed and low-speed solar wind each side of the compression region, as well as (2) the formation of forward and reverse shocks bounding the compression region (long edges of the grey area). The stream interface (SI) is identified here as the dashed line inside the CIR.

mission was designed to study solar phenomena stereoscopically and to study solar wind structures in a multi-spacecraft fashion. Here we primarily use data from the two Solar Wind Electron Analyzers (SWEA) (Sauvaud et al., 2008) and MAGnetometers (MAG) (Acuña et al., 2008) from the In-situ Measurements of PArticles and CME Transients (IMPACT) instrument suite (Luhmann et al., 2008), complemented by ion data from the PLasma And Supra-Thermal Ion Composition (PLASTIC) instruments (Galvin et al., 2008).

Identical instruments are present onboard each of the two STEREO spacecraft. The spacecraft are non-spinning due to the solar imaging requirements of the mission. The SWEA electron instruments are top-hat electrostatic analyzers with an additional deflection system (from setting a finite potential on two curved plates at its entrance) that allows measurement of incoming particles up to angles of $\pm \sim 65^{\circ}$ from the regular top-hat entrance (which looks in the plane perpendicular to the Sun-spacecraft direction), in the energy range $[\sim 0$, $2] \mathrm{keV}$. The SWEA instruments thus measure most of the electron distribution function ( $\sim 80 \%$ of the unit sphere) apart from two cones centered in the sunward and anti-sunward directions. When the magnetic field lies in these cones, pitch 
angle coverage is limited. Such data are not used for statistical analyses as explained in Sect. 3.2.1.

The data from the normal telemetry mode used here consist of 3-D distribution functions with 16 energy bins and a resolution of $\sim 22.5^{\circ}$ in azimuth and $\sim 21.7^{\circ}$ in polar angles. They are obtained from 2 second measurement intervals every $30 \mathrm{~s}$. The primary data used here are PA distributions for the energy bin centered at $\sim 250 \mathrm{eV}$. This energy is used here as it is characteristic of the suprathermal population and well above the energy where significant field-aligned anisotropies in the core distribution can affect the suprathermal PA distribution (e.g., Phillips et al., 1989; Phillips and Gosling, 1990), consistent with previous studies (cf. introduction). PA distributions have been transformed to the solar wind frame using ion flow measurements.

\section{Observations}

\subsection{Event illustration}

Figure 3 shows solar wind ion, electron and magnetic field data from ST-A (cf. caption for details) for a 3-day interval starting 6 August 2007. This interval provides an illustration of a typical CIR crossing during the STEREO era under study.

The beginning of the interval shows the period prior to the CIR. The solar wind speed and magnetic field strength were low and the strahl was first observed anti-field-aligned at PA $180^{\circ}$ (panel c). The HCS, where the magnetic field switched polarity from toward to away from the Sun, was crossed at about 04:30 UT on 6 August. After the HCS, the strahl thus became field-aligned $\left(\mathrm{PA} 0^{\circ}\right)$ for the remainder of the interval displayed. Note that the magnetic signature of the HCS becomes evident when a zoom-in with an adapted $y$-axis scale is performed (not shown). After the HCS, ST-A still observed typical slow solar wind until about 03:00 UT on 7 August when all parameters intensified, marking the entrance into the compression region. The SI was observed inside the compressed region at 07:10 UT when the ion density dropped and the temperature increased, together with a jump in solar wind speed. This boundary clearly separated two very different plasma regimes. After the SI, ST-A stayed in the compression region for a while before definitely entering the pristine high-speed solar wind stream at $\sim 14: 00 \mathrm{UT}$ on 8 August.

There were no forward or reverse shocks bounding this CIR (cf. shock list compiled by L. Jian: http://ssc.igpp.ucla. edu/forms/stereo/stereo_level_3.html), although steep magnetic field gradients can be observed on occasions (e.g., $\sim$ 04:00 UT and $\sim 20: 00$ UT on 7 August, and $\sim 06: 00$ UT on 8 August). Although shocks do develop on occasion at $1 \mathrm{AU}$ on the edges of CIRs, this event was chosen specifically because it lacks the presence of bounding shocks. Despite this absence of shocks, suprathermal electron phase space den- sities (PSDs) are strongly enhanced inside the compression region at all PAs. This provides a source of enhanced PSD for subsequent leakage, as discussed in Sect. 4.

CSEs are observed primarily on four occasions in the vicinity of this CIR (thick black arrows in panel c). The first occurrence is around $\sim 00: 00$ UT on 7 August. This corresponds to a period prior to entry into the compression region, and thus prior to the SI. The second to fourth occurrences are around 12:00 UT on 7 August, and around 02:00 and 09:00 UT on 8 August. Those intervals are inside the compression region: CSE patterns are thus not confined to regions outside CIRs. The last period of CSE pattern is observed outside the CIR between 14:00 and 18:00 UT on 8 August.

After about 18:00 UT on 8 August, the CSE pattern disappears. This disappearance corresponds to a decrease of PSD in the counter-streaming direction at $180^{\circ}$, as compared to the CSE around 09:00 UT. The enhanced PSDs measured in the counter-streaming direction (at $180^{\circ}$ ) around 09:00 UT are thus presumably due to leakage of electrons from stronger field regions of the CIR, since those are absent at larger distances away from the CIR (after 18:00 UT). In addition to enhanced PSD values at $180^{\circ}$, the PSD values are clearly depressed at $90^{\circ}$ around 09:00 UT as compared to values observed at $90^{\circ}$ after 18:00 UT on 8 August. This confirms that leakage of enhanced counter-streaming PSDs from CIRs (Gosling et al., 1993) and the process of $90^{\circ}$ halo depletion (Gosling et al., 2001a) both conspire to create strong CSE patterns in the PA spectrograms, as suggested by Steinberg et al. (2005).

\subsection{Statistical analyses of CSE}

\subsubsection{Data treatment and analysis}

We utilized all STEREO data from the period 1 March-31 December 2007. We used 10 min averages of the electron PA distributions and magnetic field calculated in the following manner. Individual PA distributions are calculated using 2 s electron distribution functions (i.e., 96 look directions: 16 azimuth and 6 polar angles) and the average magnetic field during that $2 \mathrm{~s}$ interval. Individual PA distributions used here are made of 18 bins $\left(10^{\circ}\right.$ each) from a re-sampling of the 96 PAs of each original look direction. Individual $2 \mathrm{~s} \mathrm{elec-}$ tron distribution functions are telemetered only every $30 \mathrm{~s}$. Twenty consecutive PA distributions are thus used to create the $10 \mathrm{~min}$ averages. Therefore, only magnetic field changes at scales shorter than $2 \mathrm{~s}$ during any sample may affect the resulting averaged PA distribution. For the electron data such averaging is needed because the count rate at $\sim 250 \mathrm{eV}$, in the large depletions for individual distributions, is sometimes close to the 1-count level when the ambient solar wind has a low density. The SWEA instruments do not measure in two cones $\left(25^{\circ}\right.$ half width) directed towards and away from the Sun. Therefore, all data from intervals when the magnetic 

STEREO-SWEA
$\operatorname{AHEAD}(\mathrm{SC} 1)$
$06 /$ Aug/2007

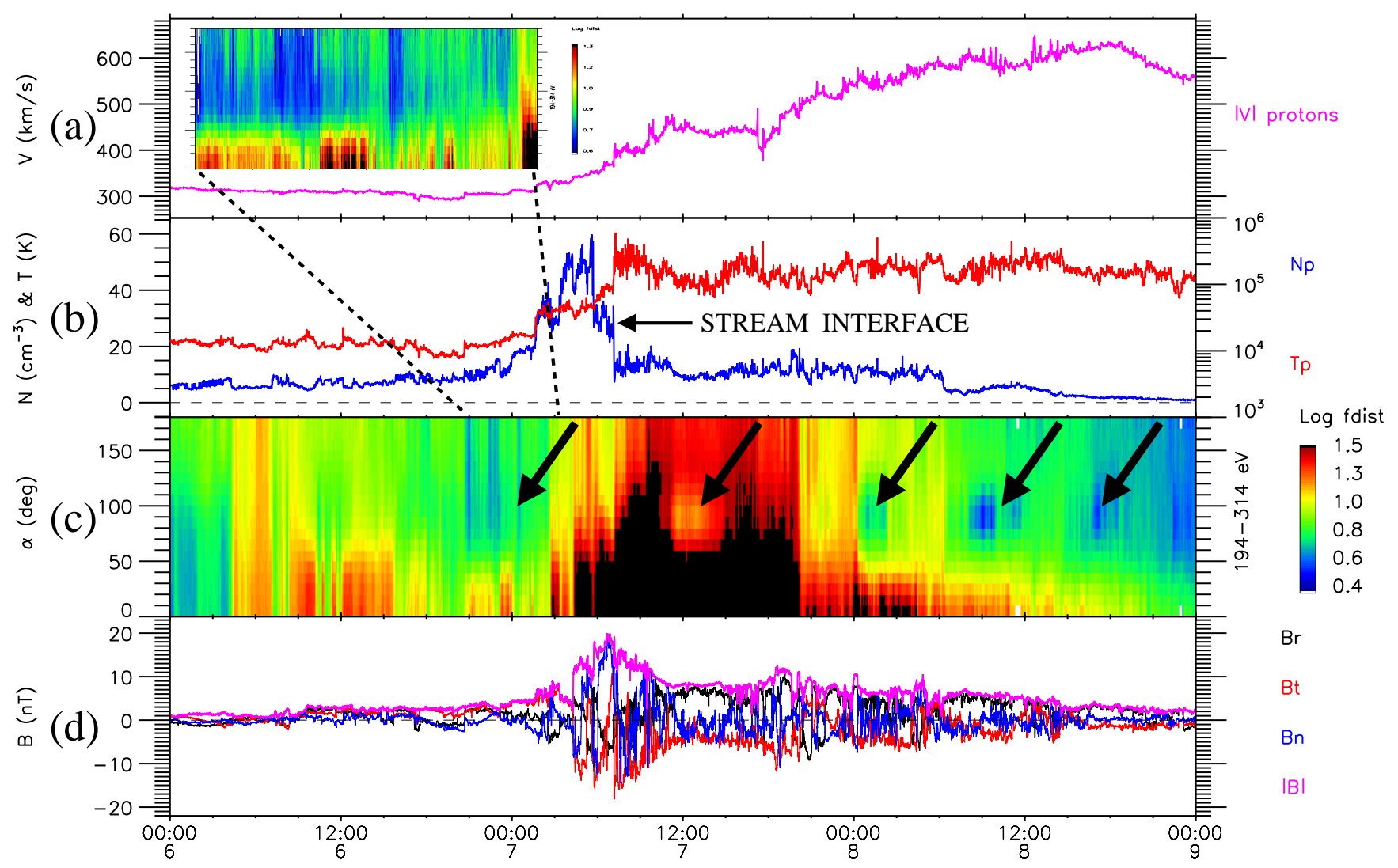

Fig. 3. Ion, electron and magnetic field data from ST-A during the passage of a CIR in August 2006 (spacecraft position was (+1973, -5271 , 45) $R_{\mathrm{E}}$ in GSE, on 7 August 2007). The panels show (a) the ion bulk speed, (b) the ion density (left y-axis) and temperature (right y-axis), (c) a pitch angle-time spectrogram for $\sim 250 \mathrm{eV}$ suprathermal electrons, and (d) the magnetic field magnitude and components in RTN. Counter-streaming suprathermal electron patterns (CSE) are observed prior to the CIR, around the beginning of 7 August 2007 (first thick black arrow), as best displayed in the zoom-in pitch angle spectrogram insert of panel (a). CSEs are also clearly observed within (second to fourth black arrow) and after the CIR (last black arrow) in the fast solar wind.

Table 1. Occurrence rate (in percent) of solar wind counterstreaming electron pitch angle distributions for each STEREO spacecraft during the solar minimum period 1 March-31 December 2007. These are given as a function of the depletion value (i.e., relative to the ambient halo value; cf. text).

\begin{tabular}{lcccc}
\hline Depletion value & 0.8 & 0.7 & 0.6 & 0.5 \\
\hline STEREO-A rate (\%) & 15.5 & 9.7 & 5.5 & 3.1 \\
STEREO-B rate (\%) & 19.7 & 13.4 & 9.1 & 5.9 \\
\hline
\end{tabular}

field had a strong radial component lead to truncated PA distributions, with a lack of data for PAs around $0^{\circ}$ and $180^{\circ}$. In our analyses we removed all such PA distributions that did not have full PA coverage (corresponding to $\sim 15 \%$ of the original dataset).
For each $10 \mathrm{~min}$ PA distribution, we collect the average PSD values from 0 to $20^{\circ}, 90 \pm 10^{\circ}$ and 160 to $180^{\circ}$. The highest value of either $0^{\circ}$ or $180^{\circ}$ is labeled as the strahl level; the lower corresponds to the halo level (Fig. 4); note this halo level may correspond to an enhanced PSD level, compared to the true halo, if strong back-scattering or heating occurs in association with CIRs. The PSD value at $90^{\circ}$ then allows us to identify a possible counter-streaming beam pattern and to quantify its magnitude. We remind here that we term CSE those PA distributions for which the field-aligned and antifield-aligned PSD are higher than at $90^{\circ}$. In the following we consider the PSD value at $90^{\circ} \mathrm{PA}$ relative to the halo PSD value as defined above. We term this the "depletion value". For instance, a depletion value of 0.8 corresponds to a $20 \%$ reduction in the $\mathrm{PSD}$ value at $90^{\circ}$ as compared to the halo value at $0^{\circ}$ or $180^{\circ}$ as the case may be. We also estimate the full width at half maximum (FWHM) of the depletions, using a polynomial fitting procedure on the full PA distribution (with 18 angular bins), for depletion values of 0.8 and lower. 


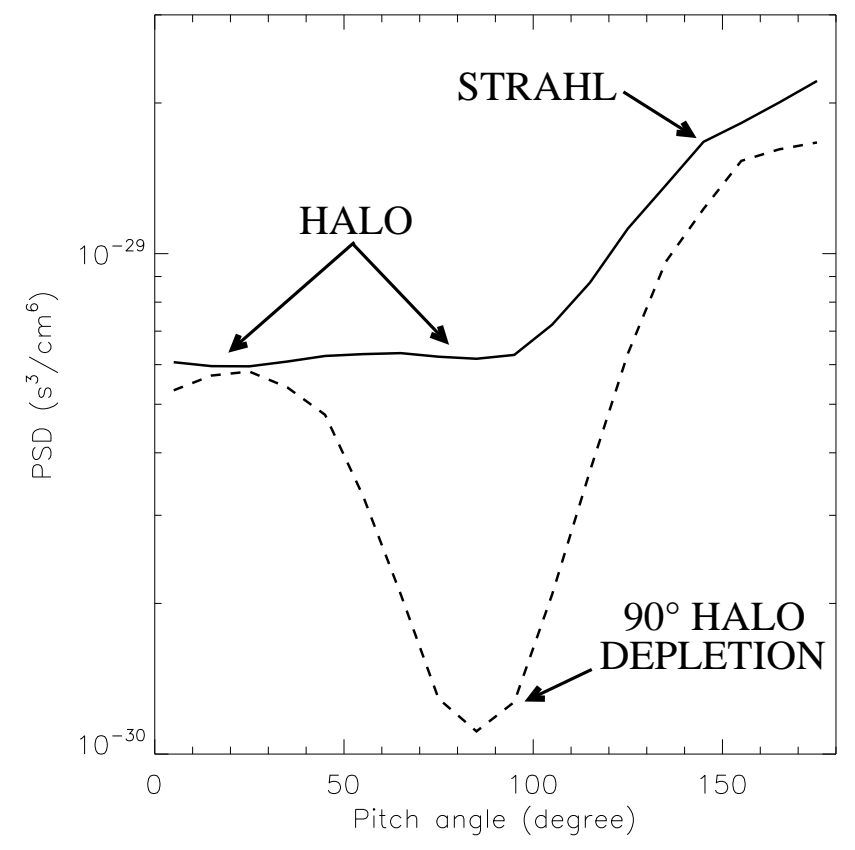

Fig. 4. Sample suprathermal electron PA spectra at $\sim 250 \mathrm{eV}$. Those are not from the event of Fig. 3, and are merely used here to exemplify the definitions used in Sect. 3.2.1.

Because our prime interest is the relationship between CSEs and CIRs, which dominantly recur during solar minimum, we removed four known ICME intervals from the analyses (list compiled by L. Jian: http://ssc.igpp.ucla.edu/forms/ stereo/stereo_level_3.html).

\subsubsection{Statistical results}

Table 1 shows the CSE occurrence rate at each spacecraft for four separate depletion value thresholds. It shows the percentage of CSE identified for the given depletion value threshold within the entire dataset of PA distributions available for analysis. The results show that stronger CSE depletions are less common, which is expected. Overall, these occurrence rates are comparable to the value of $10 \%$ estimated by Gosling et al. (2001a) from a visual survey of ACE data, and which were attributed to the process of $90^{\circ} \mathrm{PA}$ depletion described in that paper. We note from Table 1 that the percentage of CSE events observed at ST-B is systematically greater than that at ST-A, whatever the depletion value threshold. This is possibly related to ST-B being somewhat farther from the Sun, where CIRs have further developed and magnetic field strengths are lower on average.

Figure 5a shows the normalized (to the peak values) occurrence distributions of the solar wind ion bulk speed for times of CSE (solid line; depletion value threshold of 0.8) and for the whole dataset (dashed line), for ST-A and ST-B combined. This plot shows a tendency for CSE to occur in a bimodal fashion with preference for low and high speed solar wind, and a lower occurrence at intermediate speeds (400$500 \mathrm{~km} / \mathrm{s}$ ). The distribution of solar wind speeds for all data shows a predominance of slow solar wind with a monotonic gradual decrease towards fast wind.

Figure 5b shows the occurrence distributions of the magnetic field strength, normalized to the peak occurrence, for ST-A and ST-B combined. The solid line displays the occurrence distribution of magnetic field strength for times of CSE (for a depletion value threshold of 0.8 ) and the dashed line shows that for the whole dataset. Figure $5 \mathrm{~b}$ shows that the magnetic field magnitude is not significantly lower, on average, for times of CSE (solid lines) than for all data (dashed lines).

Figure 6 shows a scatter plot of the full width at half maximum (FWHM, from a polynomial fitting procedure) of the CSE PA distributions as a function of the depletion value, for ST-A and ST-B combined. It shows an increase in the width of the depletion for decreasing depletion value.

Note that the trends observed in Figs. 5 and 6 are essentially similar if plotted for each spacecraft separately or for lower depletion values (not shown).

\subsubsection{Superposed epoch analysis}

Table 2 gives times of SIs associated with CIRs during the period of interest for both ST-A and ST-B. This list was built from a visual examination of ion and magnetic field data. The selection of the SI times was based on the expectation of a density decrease, a temperature increase, a bulk speed increase, and a nearby maximum in magnetic field strength (Burlaga, 1974; Gosling et al., 1978). For an SI to be in the list, we further required that either one of the two spacecraft observed a speed enhancement larger than $200 \mathrm{~km} / \mathrm{s}$ over the inferred CIR, from the pristine slow wind to the fast wind. We estimate possible errors on these times to be of the order of a few hours. Such errors do not affect the results described later in major ways, as verified by separately using the SI list compiled by L. Jian (http://www.ssc.igpp.ucla. edu/forms/stereo/stereo_level_3.html), which only extends as of the time of writing until October 2007.

All usable data points were assigned a time relative to the closest stream interface from Table 2 for each spacecraft independently. Figure 7 shows the normalized (to peak value) CSE occurrence (solid line) as a function of epoch relative to stream interfaces (epoch zero). The dashed line shows the normalized (to peak value) occurrence for all data, i.e., with and without CSE. The dotted line shows the CSE occurrence relative to the total occurrence of data (i.e., solid curve normalized to dashed curve). Note that the temporal separations between SIs in Table 2 are widespread (from a couple days to more than 15 days). The total number of data points (dashed lines) thus falls off gradually on each side of zero epoch, and it does so in an irregular manner owing to the presence of data gaps and unused data (for example because of poor PA coverage or the presence of an ICME). 

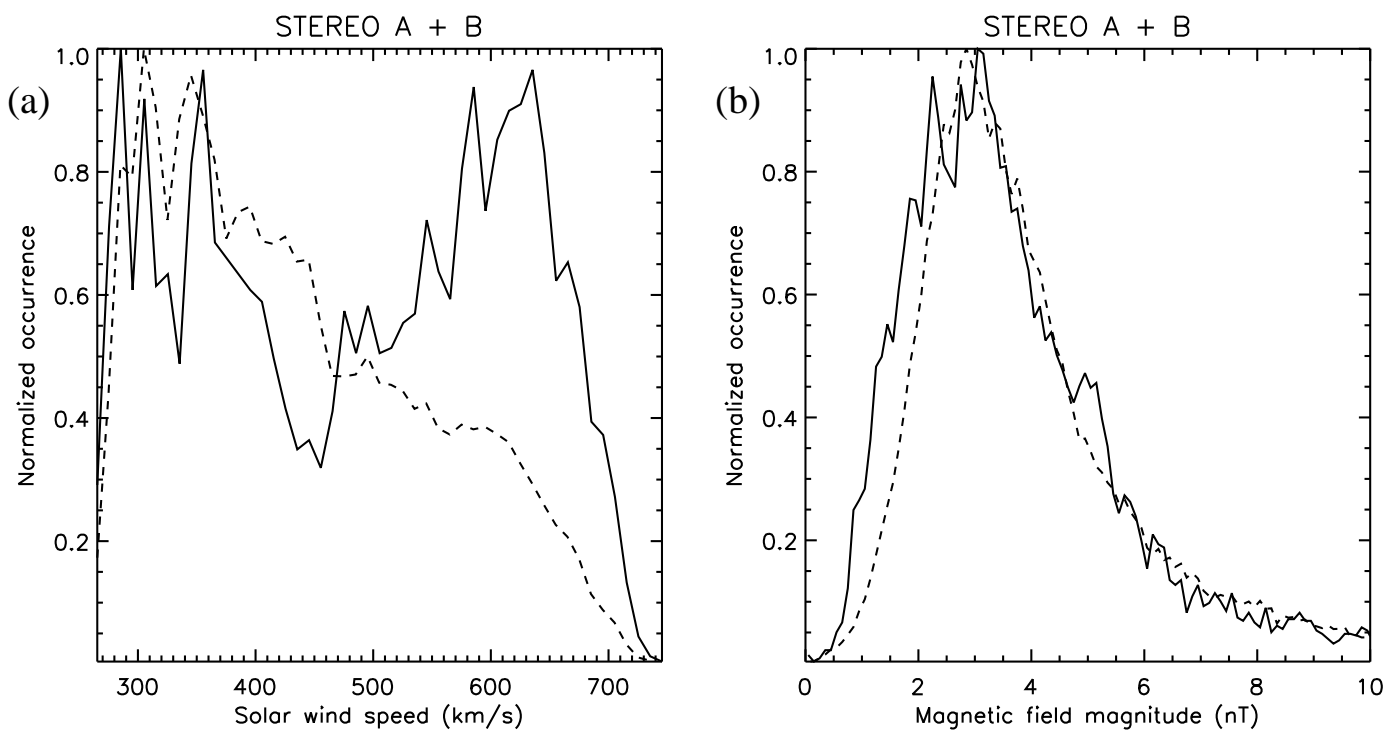

Fig. 5. Normalized occurrence distributions of (a) bulk solar wind speed, and (b) magnetic field magnitude, for data from ST-A and ST-B combined. The dashed lines show the distributions for the whole dataset used in the study, while the solid lines show the distribution for times of counter-streaming suprathermal electrons (CSE). The distributions are for a depletion value threshold of 0.8. Those for other depletion values are similar (not shown).

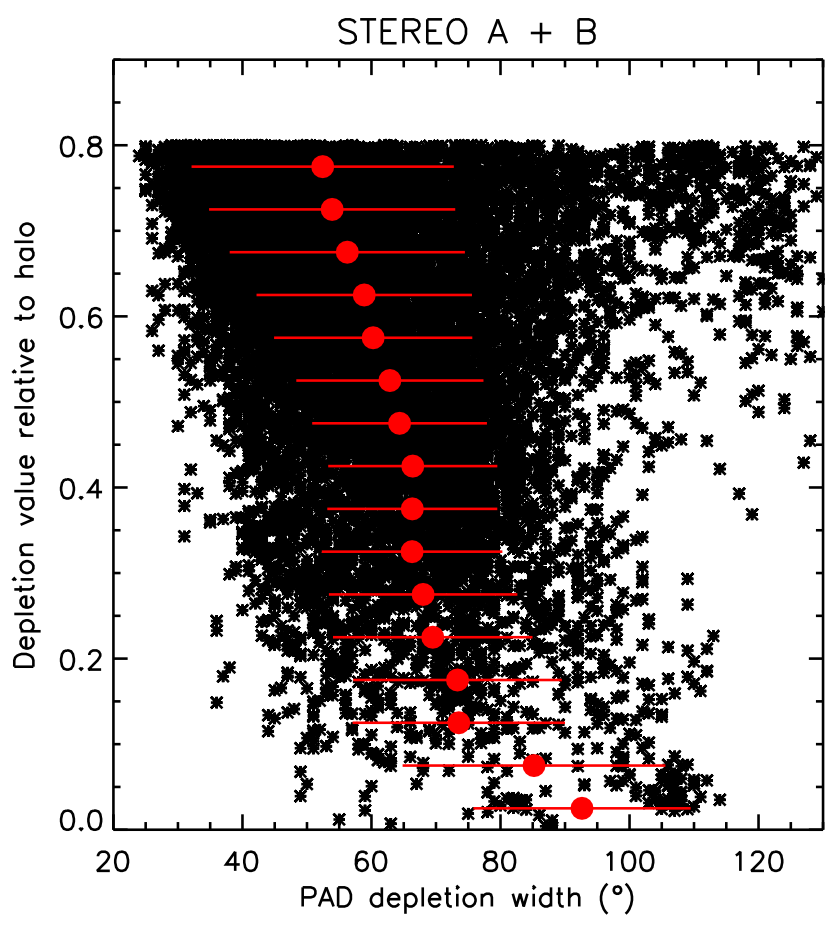

Fig. 6. Scatter plot of the full width at half maximum (FWHM) of the depletion in electron PA distributions as a function of the depletion value (cf. Fig. 3 and text for details), for ST-A and ST-B combined. Average values of the FWHM (red dots), with standard deviations (horizontal red lines), are shown as a function of the depletion value (bin size of 0.5 ).
Figure 7a shows the results for a depletion value of 0.8 , while Fig. 7b shows those for a depletion value of 0.6. Comparison of Fig. 7a and $\mathrm{b}$ illustrates that the global features do not depend on the depletion value. This fact is true for other depletion values (not shown). Results shown in later figures are also similar whatever the depletion value used (though with varying statistics). The plots displayed from now on are only for a depletion value of 0.8 .

Figure 7 demonstrates that CSEs often occur near (both before and after) the stream interfaces. There is a strong dip in CSE occurrence right at the stream interface, and the occurrence falls off at some distance each side of the interface. We also note the presence of two peaks in the pre-SI period around -1 and -2.5 days.

Figure 8 shows the superposed epoch results for various plasma parameters (cf. caption). Figure 8a shows that, on average, the strahl intensity peaked around the SI, and the strahl intensity was statistically similar between times of CSE and for all data. Figure $8 \mathrm{~b}$ shows the general behavior expected for CIRs, with a peak in magnetic field strength at the SI. The remainder of Fig. 8 displays the expected velocity increase, as well as the temperature increase while a density decrease across the SI from the slow to fast solar wind.

Figure 9 presents the occurrence distributions of the ratio of the local magnetic field strength to that at the nearby SI for times of CSE (solid lines) and for all data (dashed lines). For this analysis, the magnetic field from the nearby SI (Table 2) is determined as the strongest magnetic field in a $\pm 2 \mathrm{~h}$ window around the closest SI for any given data sample. Interestingly, it appears that during times of CSE the distribution is not statistically different from that for all data. 

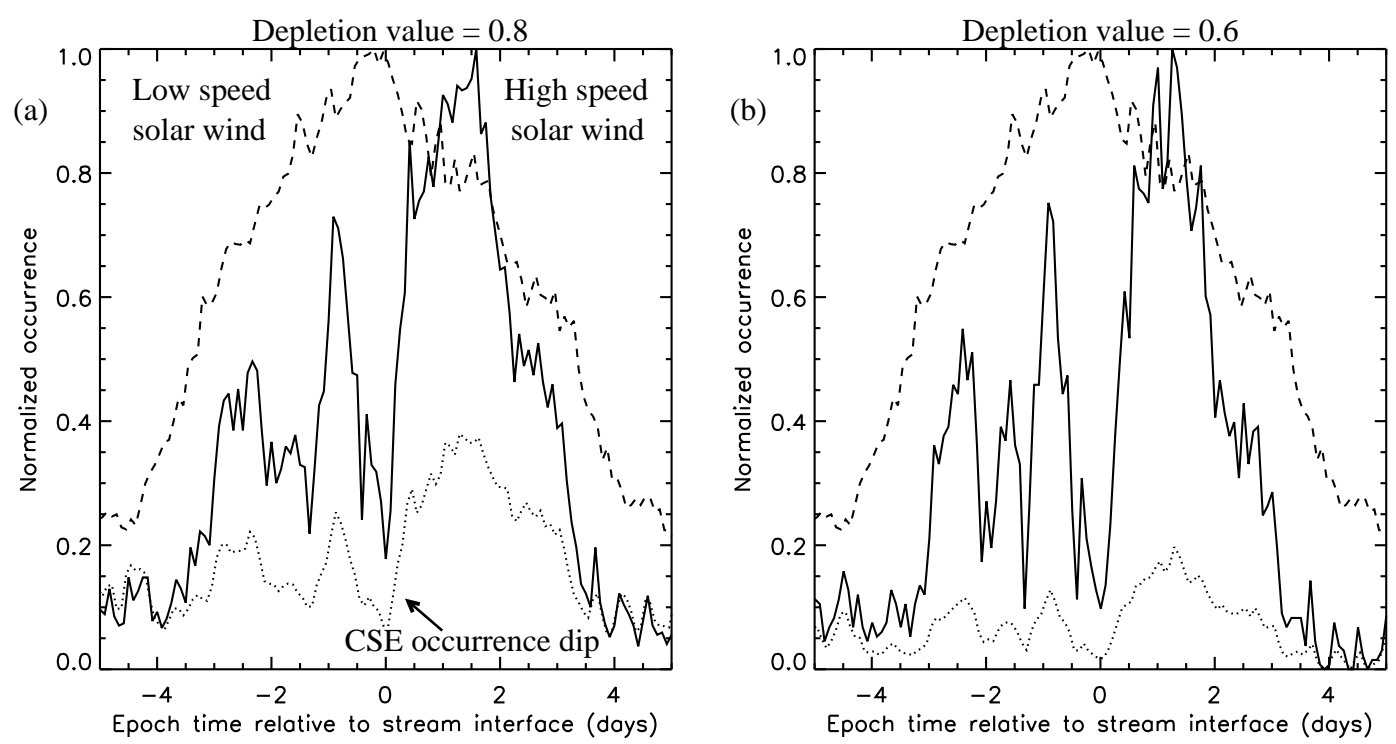

Fig. 7. Superposed epoch analysis of the occurrence of counter-streaming suprathermal electrons (CSE; solid line) and of all data (dashed line), relative to the time of the closest stream interface from Table 2, and normalized to the peak value. Epoch zero thus corresponds to the times of SI crossing, with negative (positive) epoch times being the preceding slow solar wind (following high speed wind). A two-hour interval binning has been used. The dotted lines show the CSE occurrence normalized to that of all data (i.e., solid curve normalized to dashed curve). (a) Results for a depletion value of 0.8. (b) Results for a depletion value of 0.6. The CSE occurrence in panel (a) varies between $\sim 10$ and 270 counts (per 2-h bin), giving a high statistical significance to the trends. Occurrences are about twice to three times smaller in panel (b) (cf. Table 1), which is still significant. See text for further details.

\section{Discussion}

\subsection{Role of compression, heating and leakage}

Figure 5a shows that CSE patterns occur more frequently during high-speed solar wind since the background dataset is dominated by slow solar wind. The distribution presents a bi-modal behavior with preference for either slow or highspeed solar wind. The trend of Fig. 5a is thus consistent with the superposed epoch results of Fig. 7. CSE are preferentially observed both before and after the passage of CIRs, i.e., in the slow- and high-speed winds but not near the SI where the solar wind speed is intermediate. This is as expected from the schematic of Fig. 2. At the SI in the region with enhanced magnetic field, and from the view-point of the mechanism of $90^{\circ}$ PA depletion proposed by Gosling et al. (2001a), there is no depletion, and thus no counter-streaming pattern related to it, because electrons mirror there and fill in the range of $\mathrm{PA}$ centered at $90^{\circ} \mathrm{PA}$.

Early work on the topic of CSEs in the vicinity of CIRs primarily related their occurrence to the presence of bounding shocks (Gosling et al., 1993). The ability of shocks to accelerate (heat) particles to suprathermal energy implied that such CSE patterns should primarily be observed well beyond rather than at $1 \mathrm{AU}$, because co-rotating shocks most often form after the CIR has passed $1 \mathrm{AU}$. However, Steinberg et al. (2005) noted that enhanced sunward-directed electron fluxes are frequently observed at $1 \mathrm{AU}$ as well, despite the lack of shocks locally (see also Gosling et al., 1978). From these studies it was realized that (1) the presence of CSEs in the pristine solar wind on either side of the CIR may owe to magnetic connection to shocks that have formed farther out in the heliosphere, and (2) that heating also occurs at the bounding pressure waves even when shocks have not yet developed at $1 \mathrm{AU}$.

Although no shocks bound the CIR illustrated in Fig. 3, either forward or reverse, CSEs are observed on both sides of the SI in the slow and fast winds (e.g., first and last thick arrows) and suprathermal electron PSDs are enhanced within the CIR. The latter is confirmed statistically for the strahl in Fig. 8a. The compression that results from stream interactions has early been studied through hydrodynamic and magneto-hydrodynamic modeling (Carovillano and Siscoe, 1969; Pizzo, 1978; Gosling et al., 1978). These works illustrate the fact that simple adiabatic compression leads to enhanced densities and temperatures in the compression region before shocks actually form. However, enhanced density and temperature does not correspond to enhanced suprathermal PSDs at all PAs. Simple adiabatic compression of the populations that flow into the CIR from the upstream regions (PA from $0^{\circ}$ to $90^{\circ}$ only for the event of Fig. 3) in principle (Liouville's theorem) only leads to enhanced PSDs in the perpendicular direction. In Fig. 3, for example, the enhancement in PSD at $0^{\circ}$ PA observed at $\sim 20: 00$ UT on 7 August (i.e., with upstream around 21:00 UT and downstream beforehand at 18:00 UT), which is associated with a pressure wave at the 


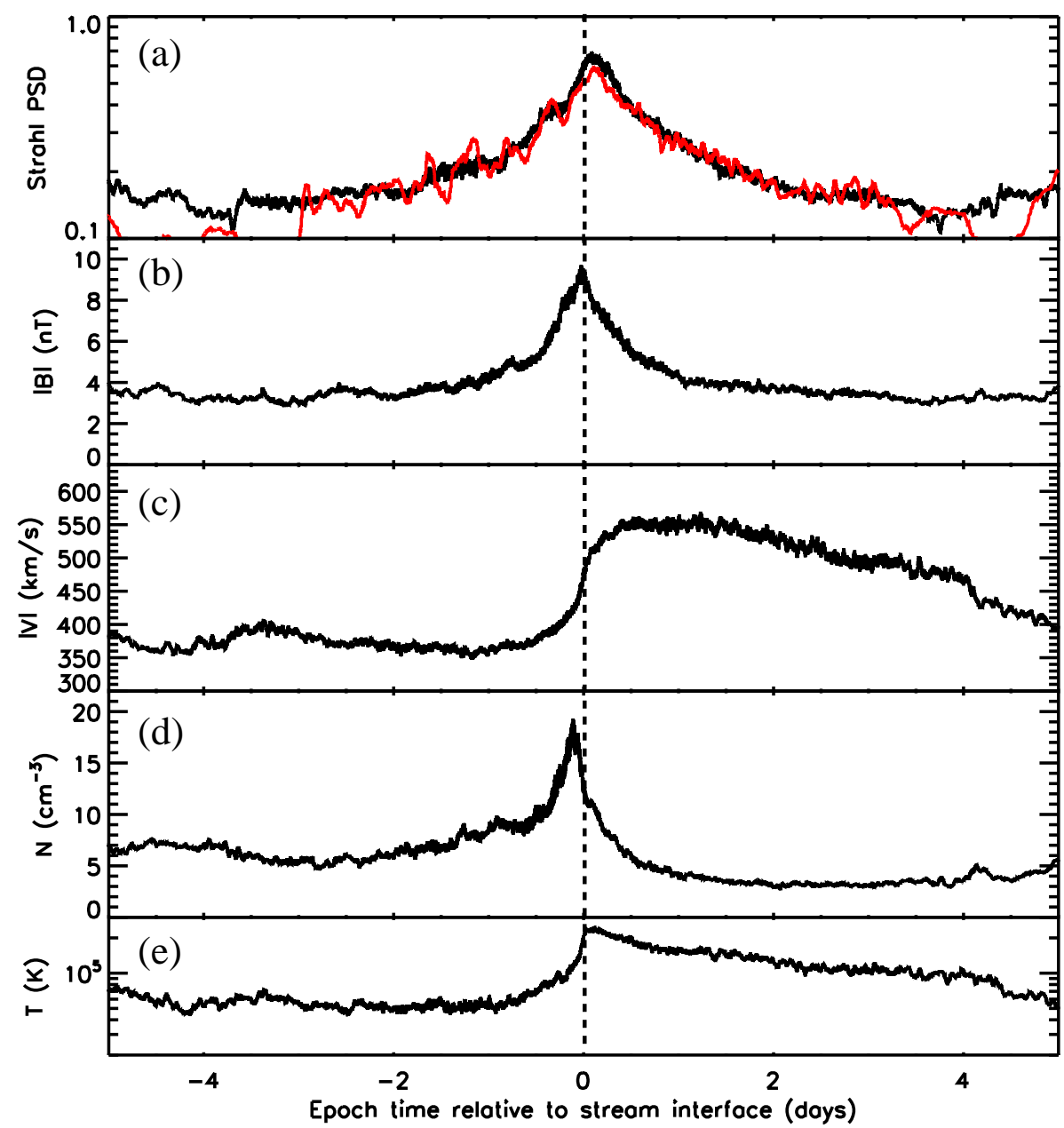

Fig. 8. Superposed epoch results from all data relative to the times of the stream interfaces of Table 2. Results are shown for (a) the strahl phase space density (arbitrary units), (b) the magnetic field magnitude, (c) the ion bulk solar wind speed, (d) the ion density, and (e) the ion temperature. In panel (a), the results for the strahl PSD during times of CSE only is also shown in red; it illustrates the fact that the strahl during CSE intervals has a similar strength, on average, as for other times. All curves are 100-point running averages of the superposed epoch dataset (cf. text for details).

rear of the CIR, can only be explained by non-adiabatic heating processes, despite the lack of a fully steepened shock. We thus concur with Steinberg et al. (2005) that additional non-adiabatic heating (such as through electro-magnetic turbulence) is required at such pressure waves. Fully developed shocks do not constitute a necessary ingredient for enhanced suprathermal electron PSDs within CIRs.

The sunward-directed (i.e., $90-180^{\circ}$ PA in Fig. 3) part of the PA distributions, which also shows anomalously enhanced PSDs inside the CIR, is likely explained by the combination of mirroring (towards the enhanced magnetic field at the SI) and PA scattering of the enhanced strahl PSDs created through heating as described above (PA scattering is further discussed in Sect. 4.3). The creation of this sunwarddirected population inside the CIRs, following the sequential heating and mirroring/scattering of the pristine anti-sunward- directed halo and strahl, provides a source of enhanced suprathermal electrons directed sunward and which may easily leak out towards upstream regions. The leaking particles then focus along the magnetic field as they exit into a lower magnetic field so as to create a CSE pattern. The notion of leakage here is not limited to leakage into the pristine regions outside the CIRs. As the event of Fig. 3 demonstrates, CSEs may form even inside the CIRs as long as the local magnetic field strength is lower than farther out along the field lines (Gosling et al., 2001a).

\subsection{Role of pitch angle scattering}

Theoretically, the scenario proposed by Gosling et al. (2001a) for the formation of PSD depletions at $90^{\circ} \mathrm{PA}$ (Fig. 1) ought to lead to complete suprathermal electron 
Table 2. List of stream interfaces associated with CIRs at each of the two STEREO spacecraft during the period 1 March-31 December 2007. See Fig. 2 and text for SI definition.

\begin{tabular}{|c|c|}
\hline $\begin{array}{c}\text { ST-A: } \\
\text { Year DOY Month Day Hour }\end{array}$ & $\begin{array}{c}\text { ST-B: } \\
\text { Year DOY Month Day Hour }\end{array}$ \\
\hline 200765030614 & 200765030616 \\
\hline 200771031212 & 200771031214 \\
\hline 200784032502 & 200784032508 \\
\hline 200791040102 & 200791040104 \\
\hline 200798040822 & 200799040910 \\
\hline 2007113042302 & 2007113042308 \\
\hline 2007117042722 & 2007117042716 \\
\hline 2007127050714 & 2007127050714 \\
\hline 2007138051816 & 2007138051808 \\
\hline 2007144052416 & 2007144052414 \\
\hline 2007155060414 & 2007155060400 \\
\hline 2007165061418 & 2007165061410 \\
\hline 2007173062206 & 2007173062206 \\
\hline 2007181063008 & 2007180062914 \\
\hline 2007185070412 & 2007184070314 \\
\hline 2007192071114 & 2007191071022 \\
\hline 2007196071506 & 2007195071412 \\
\hline 2007201072104 & 2007201072006 \\
\hline 2007208072706 & 2007207072614 \\
\hline 2007210072912 & 2007210072908 \\
\hline 2007219080708 & 2007218080612 \\
\hline 2007223081104 & 2007222081016 \\
\hline 2007238082620 & 2007237082500 \\
\hline 2007246090306 & 2007244090110 \\
\hline 2007250090722 & 2007249090614 \\
\hline 2007258091506 & 2007257091410 \\
\hline 2007264092122 & 2007263092002 \\
\hline 2007273093002 & 2007271092808 \\
\hline 2007277100408 & 2007276100302 \\
\hline 2007292101908 & 2007290101708 \\
\hline 2007299102606 & 2007297102420 \\
\hline 2007314111016 & 2007314111000 \\
\hline 2007318111410 & 2007317111308 \\
\hline 2007325112104 & 2007324112000 \\
\hline 2007330112600 & 2007327112322 \\
\hline 2007346121216 & 2007343120914 \\
\hline 2007353121900 & 2007350121612 \\
\hline 2007355122100 & 2007352121818 \\
\hline
\end{tabular}

depletions within the cone defined by the conservation of the first adiabatic invariant and centered at $90^{\circ} \mathrm{PA}$. However, the depletions are in reality never totally devoid of particles (cf. Fig. 4). As noted by Gosling et al. (2001a), this likely owes to the continuous occurrence of wave-particle interactions. The associated PA scattering progressively refills the depletions and gives them their typical reversed Gaussian shape (dashed curve in Fig. 4). Assuming a fixed PA scattering coefficient (in time and space), this refilling is expected to be more efficient (or faster) for depletions with smaller angular

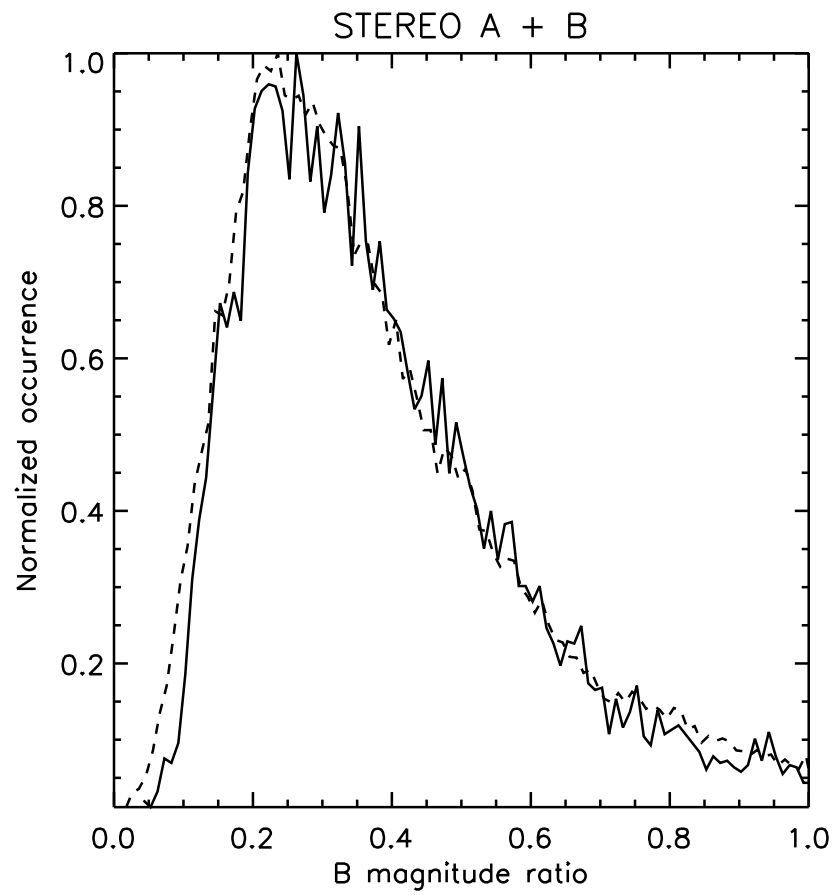

Fig. 9. Occurrence distributions of the ratio of the local magnetic field strength to that at the nearby SI for times of CSE (solid lines) and all data (dashed lines). The magnetic field at the nearby SI (Table 2 ) is determined as the strongest field in a $\pm 2 \mathrm{~h}$ interval around the SI. During times of CSE, the distribution is not statistically different from that for all data.

width, thus explaining the correlation between the depletion value and the FWHM in Fig. 6.

Our analysis showed that the local magnetic field strength is not significantly lower for CSE as compared to that for all data (Fig. 5b). CIR-related CSEs are expected to occur for lower magnetic fields, on average, since they are typically observed in the uncompressed upstream regions. For the $90^{\circ}$ PA depletion mechanism, it is the ratio of the local to maximum (farther out along the field line) magnetic field strengths that determines the width of depletions (Gosling et al., 2001a), and which in turn ought to control their occurrence. Figure 9 shows that the magnetic field strength for times of CSE is generally significantly lower than at the nearby SI where the PA distributions are expected to loose their counter-streaming shape (due to local mirroring). However, all other data that do not present a CSE character (dashed curve), in the sense of a lower PSD at $90^{\circ} \mathrm{PA}$, are statistically recorded at times of similarly low magnetic field strengths when compared to the nearby SI maximum magnetic field strength. In other words, the local magnetic field strength is often significantly lower than at the nearby SI to which it is likely connected, yet no CSE pattern resulting from $90^{\circ} \mathrm{PA}$ depletion is observed. A likely explanation here as well is the continuous occurrence of wave-particle interactions in the vicinity of and within the CIR, with associated PA scattering refilling the depletion cone. 
Another possibility is that the Sunward-directed leaking population may sometimes be too weak in CIRs to form CSEs in the upstream regions. However, PSD values are statistically much higher inside the CIR than outside (Fig. 8a), and this is true for the sunward-directed suprathermal population, indicating that there usually exists a significant seed population for leakage. This is observed in Fig. 3c, for instance, where we further note that enhanced Sunwarddirected electron PSDs are measured on numerous occasions together with a lack of depletion at $90^{\circ} \mathrm{PA}$ (i.e., times in between the black arrows).

At some distance from the SI the magnetic topology of Fig. 2 implies magnetic connection to a CIR/SI farther out in the heliosphere, where the field strength within the CIR usually should be lower than at $1 \mathrm{AU}$. The amplitude of the ratio displayed in Fig. 9 would in principle need to be scaled accordingly. However, we believe that scaling this ratio to the actual magnetic field strength farther out (and which is unknown) is statistically unlikely to alter the fact that the two distributions in Fig. 9 are similar.

The facts (1) that $90^{\circ}$ PA depletions are never totally devoid of particles and (2) that CSEs are not observed as often as one would expect from simple magnetic field ratio arguments in the vicinity of CIRs, both suggest strong and continuous PA scattering in the vicinity of CIRs. This inferred strong PA scattering is further necessary in the scenario proposed in Sect. 4.1, where we suggest that the enhanced sunward-directed portion of the suprathermal electron distributions within the CIR stems from the combination of mirroring and PA scattering of the enhanced (compressed and heated) anti-sunward-directed strahl in the CIR. Future work ought to study the presence of appropriate waves in the vicinity of CIRs and their relation with the observation of CSEs (e.g., Horbury and Schmidt, 1999).

\subsection{Role of the bounding shocks}

Compression and heating occur within CIRs even in the absence of bounding shocks. This process, together with a necessary PA scattering process, constitutes the means to produce enhanced sunward-directed PSDs. Here "enhanced" means PSDs that are larger than the (also) sunward-directed halo PSDs observed a good distance away from the CIR, when no CSE pattern is observed (e.g., at the very end of the interval shown in Fig. 3). However, as shown by Steinberg et al. (2005) and found in the STEREO data as well (not shown), the counter-streaming sunward-directed PSDs outside the CIR-bounding shocks sometimes exceed those observed just inside (downstream), which is deemed to be the source population. In future work, we will show how the presence of shocks may indeed permit a further increase in the CSE population PSDs by means of shock reflection.

The occurrence of CSE appears to be strongly related to the presence of CIRs and shocks during solar minimum. The fact that their presence is a key ingredient, i.e., in order to provide enhanced PSDs in the sunward direction, is a currently debated theme (cf. introduction). An alternate, or complementary, mechanism is the sole occurrence of backscattering owing to wave-particle interactions (cf. introduction). As noted by Maksimovic et al. (2005), CIR- and shock-related CSE should be limited to regions of the solar ecliptic where CIRs typically form. It would be of interest in the future, using for instance data from the Ulysses spacecraft, to determine whether CSEs occur as often (as a function of their magnitude) at higher heliospheric latitudes, since the scenario depicted here would seem to preclude it if the field lines to not wander substantially in latitude (cf. Gosling et al., 2001b).

\subsection{Role of small-scale transients in the slow wind}

In the context of the present study, it is necessary to note that a non-negligible fraction of CSE patterns observed prior to CIRs likely comes from the passage of small-scale transients with closed magnetic field topologies. Indeed, recent studies have shown that transients with CSE patterns are often observed in the slow solar wind (Kilpua et al., 2009; Rouillard et al., 2009, 2010a, b) ahead of CIRs, while not in their trailing part, compatible with their inferred streamer belt origin (Wang et al., 2000; Zurbuchen et al., 2001; Crooker et al., 2004). The determination of their exact contribution to the occurrence rate of CSEs in the slow wind prior to CIRs is a topic for future studies.

\section{Conclusions}

We have studied the properties of counter-streaming $250 \mathrm{eV}$ suprathermal electron (CSE) patterns through statistical analyses of the STEREO PA distributions during the solar minimum period 1 March-31 December 2007. Our purpose was to (1) quantify the occurrence rate of CSE, (2) determine the CSE occurrence rate as a function of location relative to CIRs, and (3) investigate the potential roles of pitch angle scattering and of the presence of CIR-bounding shocks. The primary results are as follows.

1. CSE occurrence rates are of the order of $15-20 \%$ around $1 \mathrm{AU}$, compatible with previous studies. The rates of course depend on the definition criteria. There is a clear tendency to observe more CSE at ST-B, which is farther from the Sun.

2. CSEs occur for magnetic field strengths that are significantly lower than at the nearby stream interface (SI). However, CSEs are not observed as frequently as one would expect based on the assumption that the ratio of the local magnetic strength to that at the nearby SI is the only ingredient needed for their appearance. 
3. Larger CSE $90^{\circ} \mathrm{PA}$ depletion values correspond to wider depletions in pitch angles. The depletions are never totally devoid of electrons.

4. CSEs with enhancements in suprathermal electron PSDs are frequently observed on both sides of CIRs, even in the absence of bounding shocks. Enhanced PSDs in the sunward direction are observed even in the absence of CSE: the absence of CSE is not typically the result of a lack of seed population (leaking from the CIR).

5. As demonstrated with the event of Fig. 3, CSEs may form even inside CIRs as long as the local magnetic field strength is lower than farther out along the field lines.

6. CSEs are overall more frequent in the high-speed solar wind, with a bi-modal shape favoring both slow and fast wind.

7. Consistent with the latter, superposed epoch analyses demonstrate that CSE occur in both the slow solar wind prior to the SI, as well as in the high-speed solar wind after the SI. There is a significant minimum occurrence at the SI itself. CSE occurrence rate peaks above 35\% in the trailing part of CIRs.

Results (1), (2) and (3) above are suggestive of frequent, almost total, refilling of the expected depletions at $90^{\circ} \mathrm{PA}$ by wave-particle interactions and associated PA scattering. PA scattering is thus expected to be particularly strong in the CIR region. Consistently, scattering inside the CIR is required in our scenario in order to create the sunward-directed portion of the PA distribution that constitutes the source (through leakage) of the sunward directed beam in the CSE patterns observed upstream of CIRs. Non-adiabatic heating is required, in addition to scattering, to explain the enhanced PSDs inside CIRs and in the CSEs. This required additional heating/acceleration occurs whether fully developed bounding shocks are present or not (results 4 and 5).

Results (6) and (7) are consistent with the expected properties and magnetic topology in the vicinity of CIRs, as sketched in Figs. 1 and 2. CSEs are deemed to result primarily from leakage of enhanced backscattered suprathermal electrons from the CIRs, together with the process of $90^{\circ} \mathrm{PA}$ depletion owing to mirroring and focusing effects (Gosling et al., 2001a, 2002; Steinberg et al., 2005; Skoug et al., 2006). CSE events unrelated to closed magnetic field topologies are thus common in the solar wind during solar minimum, in particular in the high-speed trailing part of CIRs. It should be noted, however, that small-scale transients with closed magnetic topology likely also contribute to the detection of CSEs in the interval studied, but only in the slow solar wind prior to the SI.
Because suprathermal electrons are widely used tracers of heliospheric magnetic topology and energetic phenomena, future work ought to focus on characterizing the nonadiabatic heating mechanism that occurs within CIRs (as well as at the bounding shocks), as well as the origin and implications of pitch-angle scattering.

Acknowledgements. The authors thank the entire IMPACT and PLASTIC teams for their efforts in the development of the instruments and in the generation of the datasets. We are grateful for the use the AMDA tool at CDPP (http://cdpp-amda.cesr.fr/) which allowed easy search and visualization of CIR crossings. The authors also thank the reviewers for their constructive comments.

Topical Editor R. Forsyth thanks S. Kahler and another anonymous referee for their help in evaluating this paper.

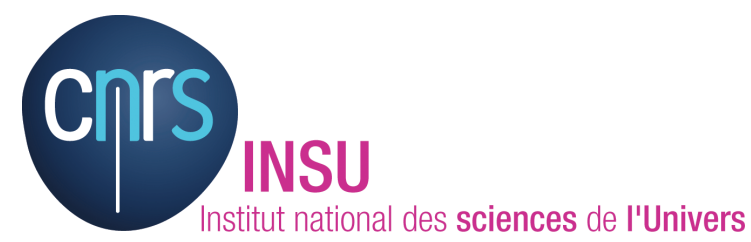

The publication of this article is financed by CNRS-INSU.

\section{References}

Acuña, M. H., Curtis, D., Scheifele, J. L., Russell, C. T., Schroeder, P., Szabo, A., and Luhmann, J. G.: The STEREO/IMPACT magnetic field experiment, Space Sci. Rev., 136(1-4), 203-226, 2008.

Burlaga, L. F.: Interplanetary stream interfaces, J. Geophys. Res., 79(25), 3717-3725, 1974.

Burlaga, L. F., Mish, W. H., and Whang, Y. C.: Coalescence of recurrent streams of different sizes and amplitudes, J. Geophys. Res., 95(A4), 4247-4255, 1990.

Carovillano, R. L. and Siscoe, G. L.: Corotating structure in the solar wind, Solar Phys., 8(2), 401-414, 1969.

Crooker, N. U., Huang, C.-L., Lamassa, S. M., Larson, D. E., Kahler, S. W., and Spence, H. E.: Heliospheric plasma sheets, J. Geophys. Res., 109, A03107, doi:10.1029/2003JA010170, 2004..

de Koning, C. A., Gosling, J. T., Skoug, R. M., and Steinberg, J. T.: Energy dependence of electron pitch angle distribution widths in solar bursts, J. Geophys. Res., 112, A04101, doi:10.1029/2006JA011971, 2007.

Feldman, W. C., Asbridge, J. R., Bame, S. J., Montgomery, M. D., and Gary, S. P.: Solar wind electrons, J. Geophys. Res., 80(31), 4181-4196, 1975.

Galvin, A. B., Kistler, L. M., Popecki, M. A., Farrugia, C. J., et al.: The plasma and suprathermal ion composition (PLASTIC) investigation on the STEREO observatories, Space Sci. Rev., 136(14), 437-486, 2008.

Gary, S. P., Scime, E. E., Phillips, J. L., and Feldman, W. C.: The whistler heat flux instability: Threshold conditions in the solar wind, J. Geophys. Res., 99, 23391-23399, 1994.

Gosling, J. T., Asbridge, J. R., Bame, S. J., and Feldman, W. C.: Solar wind stream interfaces, J. Geophys. Res., 83(A4), 14011412, 1978. 
Gosling, J. T., Baker, D. N., Bame, S. J., Feldman, W. C., Zwickl, R. D., and Smith, E. J.: Bidirectional solar wind electron heat flux events, J. Geophys. Res., 92(A8), 8519-8535, 1987.

Gosling, J. T.: Coronal mass ejections and magnetic flux ropes in interplanetary space, in: Physics of Magnetic Flux Ropes, Geophys. Monograph, No. 58, edited by: Russell, C. T., Priest, E. R., and Lee, L. C., AGU, p. 343-364, 1990.

Gosling, J. T., Bame, S. J., Feldman, W. C., McComas, D. J., Phillips, J. L., and Goldstein, B. E.: Counterstreaming suprathermal electron events upstream of corotating shocks in the solar wind beyond $\sim 2$ AU: Ulysses, Geophys. Res. Lett., 20(21), 2335-2338, 1993.

Gosling, J. T. and Pizzo, V. J.: Formation and evolution of corotating interaction regions and their three dimensional structure, Space Sci. Rev., 89(1-2), 21-52, 1999.

Gosling, J. T., Skoug, R. M., and Feldman, W. C.: Solar wind electron halo depletions at $90^{\circ}$ pitch angle, Geophys. Res. Lett., 28(22), 4155-4158, 2001a.

Gosling, J. T., McComas, D. J., Skoug, R. M., and Forsyth, R. J.: Stream interaction regions at high heliographic latitudes during Ulysses' second polar orbit, Space Sci. Rev., 97(1-4), 189-192, 2001b.

Gosling, J. T., Skoug, R. M., Feldman, W. C., and McComas, D. J.: Symmetric suprathermal electron depletions on closed field lines in the solar wind, Geophys. Res. Lett., 29(12), 1573, doi:10.1029/2001GL013949, 2002.

Gosling, J. T., McComas, D. J., Skoug, R. M., and Smith, C. W.: Magnetic reconnection at the heliospheric current sheet and the formation of closed magnetic field lines in the solar wind, Geophys. Res. Lett., 33, L17102, doi:10.1029/2006GL027188, 2006.

Hammond, C. M., Feldman, W. C., McComas, D. J., Phillips, J. L., and Forsyth, R. J.: Variation of electron-strahl width in the highspeed solar wind: Ulysses observations, Astron. Astrophys., 316, 350-354, 1996.

Horbury, T. S. and Schmidt, J. M.: Development and effects of turbulence in connection with CIRs, Space Sci. Rev., 89(1-2), 6176, 1999.

Hundhausen, A. J.: Nonlinear model of high-speed solar wind streams, J. Geophys. Res., 78(10), 1528-1542, 1973.

Kaiser, M. L., Kucera, T. A., Davila, J. M., St. Cyr, O. C., Guhathakurta, M., and Christian, E.: The STEREO mission: An introduction, Space Sci. Rev., 136(1-4), 5-16, 2008.

Kahler, S. and Lin, R. P.: The determination of interplanetary magnetic field polarities around sector boundaries using $\mathrm{E}>2 \mathrm{keV}$ electrons, Geophys. Res. Lett., 21(15), 1575-1578, 1994.

Kilpua, E. K. J., Luhmann, J. G., Gosling, J., et al.: Small solar wind transients and their connection to the large-scale coronal structure, Solar Phys., 256(1-2), 327-344, 2009.

Lavraud, B., Thomsen, M. F., Lefebvre, B., et al.: Evidence for newly closed magnetosheath field lines at the dayside magnetopause under northward IMF, J. Geophys. Res., 111, A05211, doi:10.1029/2005JA011266, 2006.

Lavraud, B., Gosling, J. T., Rouillard, A. P., et al.: Observations of a complex solar wind reconnection exhaust from spacecraft separated by over 1800 RE, Solar Phys., 256(1-2), 379-392, doi:10.1007/s11207-009-9341-x, 2009.

Lie-Svendsen, Ø., Hansteen, V. H., and Leer, E.: Kinetic electrons in high-speed solar wind streams: Formation of high-energy tails, J. Geophys. Res., 102(A3), 4701-4718, 1997.
Luhmann, J. G., Curtis, D. W., Schroeder, P., et al.: STEREO IMPACT investigation goals, measurements, and data products overview, Space Sci. Rev., 136(1-4), 117-184, 2008.

Maksimovic, M., Zouganelis, I., Chaufray, J. Y., Issautier, K., et al.: Radial evolution of the electron distribution functions in the fast solar wind between 0.3 and 1.5 AU, J. Geophys. Res., 110, A09104, doi:10.1029/2005JA011119, 2005.

Neugebauer, M. and Snyder, C. W.: Mariner 2 observations of the solar wind 1. Average properties, J. Geophys. Res., 71(19), 4469-4484, 1966.

Onsager, T. G., Scudder, J. D., Lockwood, M., and Russell, C. T.: Reconnection at the high latitude magnetopause during northward interplanetary magnetic field conditions, J. Geophys. Res., 106, 25467-25488, 2001.

Owens, M. J., Crooker, N. U., and Schwadron, N. A.: Suprathermal electron evolution in a Parker spiral magnetic field, J. Geophys. Res., 113, A11104, doi:10.1029/2008JA013294, 2008.

Phillips, J. L., Gosling, J. T., McComas, D. J., Bame, S. J., and Gary, S. P.: Anisotropic thermal electron distributions in the solar wind, J. Geophys. Res., 94, 6563-6579, 1989.

Phillips, J. L. and Gosling, J. T.: Radial evolution of suprathermal electron distributions due to expansion and collisions, J. Geophys. Res., 95, 4217-4228, 1990.

Pierrard, V., Maksimovic, M., and Lemaire, J.: Electron velocity distribution functions from the solar wind to the corona, J. Geophys. Res., 104(A8), 17021-17032, 1999.

Pilipp, W. G., Miggenrieder, H., Montgomery, M. D., Mühlhäuser, K.-H., Rosenbauer, H., and Schwenn, R.: Characteristics of electron velocity distribution functions in the solar wind derived from the Helios plasma experiment, J. Geophys. Res., 92(A2), 10751092, 1987a.

Pilipp, W. G., Miggenrieder, H., Montgomery, M. D., Mühlhäuser, K.-H., Rosenbauer, H., and Schwenn, R.: Unusual electron distribution functions in the solar wind derived from the Helios plasma experiment: Double-strahl distributions and distributions with an extremely anisotropic core, J. Geophys. Res., 92(A2), 1093-1101, 1987b.

Pizzo, V.: A three-dimensional model of corotating streams in the solar wind, 1. Theoretical foundations, J. Geophys. Res., 83(A12), 5563-5572, 1978.

Rouillard, A. P., Savani, N., Davies, J. A., et al.: A multi-spacecraft analysis of a small-scale transient entrained by solar wind streams, Solar Phys., 256(1-2), 307-326, doi:10.1007/s11207009-9329-6, 2009.

Rouillard, A. P., Lavraud, B., Davies, J. A., et al.: Intermittent release of small-scale transients in the slow solar wind: II, In-situ evidences, J. Geophys. Res., in press, doi:10.1029/2009JA014472, 2010a.

Rouillard, A. P., Davies, J. A., Lavraud, B., et al.: Intermittent release of small-scale transients in the slow solar wind: I, Remote sensing observations, J. Geophys. Res., in press, doi:10.1029/2009JA014471, 2010b.

Sarabhai, V.: Some consequences of nonuniformity of solar wind velocity, J. Geophys. Res., 68(5), 1555-1557, 1963.

Sauvaud, J.-A., Larson, D., Aoustin, C., et al.: The IMPACT Solar Wind Electron Analyzer (SWEA), Space Sci. Rev., 136(1-4), 227-239, 2008.

Saito, S. and Gary, S. P.: Whistler scattering of suprathermal electrons in the solar wind: Particle-in-cell simulations, J. Geophys. 
Res., 112, A06116, doi:10.1029/2006JA012216, 2007.

Schulz, M.: Interplanetary sector structure and the heliomagnetic equator, Astrophys. Space Sci., 24, 371-383, 1973.

Skoug, R. M., Gosling, J. T., McComas, D. J., Smith, C. W., and $\mathrm{Hu}, \mathrm{Q}$.: Suprathermal electron $90^{\circ}$ pitch angle depletions at reverse shocks in the solar wind, J. Geophys. Res., 111, A01101, doi:10.1029/2005JA011316, 2006.

Steinberg, J. T., Gosling, J. T., Skoug, R. M., and Wiens, R. C.: Suprathermal electrons in high-speed streams from coronal holes: Counterstreaming on open field lines at $1 \mathrm{AU}, \mathrm{J}$. Geophys. Res., 110, A06103, doi:10.1029/2005JA011027, 2005.

Štverák, Š., Maksimovic, M., Trávnícek, P. M., Marsch, E., Fazakerley, A. N., and Scime, E. E.: Radial evolution of nonthermal electron populations in the low-latitude solar wind: Helios, Cluster, and Ulysses Observations, J. Geophys. Res., 114, A05104, doi:10.1029/2008JA013883, 2009.
Vocks, C., Salem, C., Lin, R. P., and Mann, G.: Electron halo and strahl formation in the solar wind by resonant interaction with whistler waves, Astrophys. J., 627, 540-549, doi:10.1086/430119, 2005.

Wang, Y.-M., Sheeley Jr., N. R., Socker, D. G., Howard, R. A., and Rich, N. B.: The dynamical nature of coronal streamers, J. Geophys. Res., 105(A11), 25133-25142, 2000.

Winterhalter, D., Smith, E. J., Burton, M. E., Murphy, N., and McComas, D. J.: The heliospheric plasma sheet, J. Geophys. Res., 99(A4), 6667-6680, 1994.

Zurbuchen, T. H., Hefti, S., Fisk, L. A., Gloeckler, G., Schwadron, N. A., Smith, C. W., Ness, N. F., Skoug, R. M., McComas, D. J., and Burlaga, L. F.: On the origin of microscale magnetic holes in the solar wind, J. Geophys. Res., 106(A8), 16001-16010, 2001.

Zwickl, R. D., Asbridge, R. J., Bame, S. J., Feldman, W. C., Gosling, J. T., and Smith, E. J.: Plasma properties of driver gas following interplanetary shocks observed by ISEE-3, Solar Wind Five, NASA Conf. Publ. CP-2280, 711-717, 1983. 